\title{
Ökologischer Imperativ, Nachhaltigkeit, Planetare Grenzwerte und „One Health“ -Zielfunktionen für ein zukunftsfähiges Geoengineering
}

\section{Volker Mosbrugger}

\section{Zusammenfassung}

Der Mensch greift seit jeher in das Systemgefüge der Erde ein, doch seit dem Neolithikum entwickeln sich diese Eingriffe zunehmend zu einer nicht nachhaltigen Ausbeutung der Natur. Durch die gravierenden rasanten Veränderungen im Anthropozän wird ein modernes Erdsystem-Management oder Geoengineering, das die „Permanenz echten menschlichen Lebens“ (Hans Jonas) sichert, nun $\mathrm{zu}$ einer dringenden Herausforderung. Dafür müssen zunächst gültige Zielgrößen definiert werden, die fortlaufend immer wieder im wechselseitigen Abgleich der aus dem Management-System resultierenden Folgen angepasst werden müssen. Für ein solches weltweit wirkendes System sind sowohl technische als auch ökonomische und politische Werkzeuge notwendig, die durch eine umfassende Governance gesteuert werden.

Inzwischen leben rund 7,8 Mrd. Menschen auf dieser Erde (Mai 2020) und dennoch ging es der Menschheit insgesamt noch nie so gut wie heute: noch nie konnten so viele Menschen ohne Hunger leben, noch nie sind so viele Menschen so alt geworden und noch nie hatten so viele Menschen, und insbesondere auch Frauen, Zugang zu Bildung. Diese insgesamt positive Entwicklung basiert auf einer systematischen Ausbeutung der Natur, die alles andere als zukunftsfähig oder nachhaltig ist. Als Folge davon stehen wir heute vor einem planetaren Problemsyndrom, wie etwa Klimawandel, Biodiversitätsverlust, Entwaldung, Luftund Umweltverschmutzung, Versauerung und Überfischung der Ozeane - mit

\footnotetext{
V. Mosbrugger $(\bowtie)$

Senckenberg Gesellschaft für Naturforschung in Frankfurt am Main, Frankfurt am Main, Deutschland

E-Mail: volker.mosbrugger@senckenberg.de
} 
der Konsequenz, dass nach Schätzungen der Weltbank (Rigaud et al. 2018) im Jahr 2050 über 140 Mio. Menschen allein aus Südasien, Sub-Sahara-Afrika und Lateinamerika als Umweltflüchtlinge aus ihrer Heimat vertrieben werden.

Wie kann diese riesige Herausforderung des Anthropozäns, die Lösung der planetaren Umweltprobleme und der Übergang zu einem nachhaltigen Umgang mit dem Planeten Erde, bewältigt werden? Technisch gesprochen, liegt die Lösung in einem Erdsystem-Management oder Geoengineering, also in einer systemischen, und nicht segmentalen Behandlung der verschiedenen Umweltprobleme, da diese untereinander gekoppelt sind und letztlich alle ihre Ursache in der tragedy of the commons, in der Übernutzung der natürlichen Gemeingüter haben. Dabei sind die Begrifflichkeiten Erdsystem-Management oder Geoengineering durchaus skalierbar zu verstehen und können die lokale, regionale, kontinentale oder globale Skala betreffen; entscheidend ist jedoch, dass bei allen, auch lokalen, Eingriffen in das Erdsystem die globalen Auswirkungen und Rückkopplungen mit berücksichtigt werden.

Tatsächlich betreiben wir Menschen schon seit langem ein - bisher leider recht ignorantes - Erdsystem-Management oder Geoengineering, allerdings ohne uns dessen bewusst zu sein. Man denke nur an die großräumige Entwaldung des Mittelmeerraumes durch die Römer, die tief greifende Umgestaltung unserer Küsten und Flüsse oder die weltweite Veränderung der Biosphäre durch Landwirtschaft und Deforestation. Unser bisheriges Erdsystem-Management erfolgte ganz offensichtlich ohne ein Systemverständnis und ohne Rücksicht auf die negativen systemischen Folgeerscheinungen - die oben genannten planetaren Umweltprobleme sind die Folge davon.

Die Herausforderung für die Zukunft liegt also darin, ein skalierbares (lokales bis globales) Erdsystem-Management mit Sinn und Verstand zu entwickeln, das auf einem umfassenden Systemverständnis basiert und nicht nur auf kurzfristige Vorteile, sondern auf eine zukunftsfähige Entwicklung unseres Planeten zum Wohle aller Menschen abzielt. Was also sind dann die Zielgrößen eines derartigen Geoengineering? Eine Zielgröße sollte der Ökologische Imperativ des Philosophen Hans Jonas (1903-1993) sein: „Handle so, dass die Wirkungen deiner Handlungen verträglich sind mit der Permanenz echten menschlichen Lebens auf Erden." (ebd. 1984, S. 36) Als übergeordnete Maxime ist diese Anforderung ohne Zweifel wichtig, sie wird aber bei konkreten Umwelteingriffen nur selten entscheidungsleitend sein können.

Eine weitere Zielgröße sollte die Nachhaltigkeit sein, wie sie im Brundtlandt Report definiert wurde: „Sustainable development meets the needs of the present without compromising the ability of future generations to meet their own needs.“ (ebd. 1987, S. 16) Inzwischen besteht Konsens, dass diese Nachhaltigkeit 
zumindest drei Dimensionen umfasst: eine soziale Dimension (people), eine ökonomische Dimension (prosperity) und eine ökologische Dimension (planet). Eine echte Nachhaltigkeit im Sinne einer umfassenden Zukunftsfähigkeit muss alle drei Dimensionen gleichermaßen im Blick behalten und entsprechend zukunftsfähig gestalten. Doch haben über die Jahrhunderte ganz offensichtlich die sozialen und ökonomischen Interessen über die ökologischen dominiert, sodass die ökologischen Herausforderungen inzwischen ernsthafte Probleme auch für die soziale und wirtschaftliche Entwicklung bedeuten - dieses Ungleichgewicht muss künftig durch ein Erdsystem-Management überwunden werden.

Eine weitere Zielgröße für ein modernes Erdsystem-Management könnten auch die von Johan Rockström und Kollegen eingeführten Planetaren Grenzwerte (planetary boundary conditions) sein. In einflussreichen Veröffentlichungen (Rockström et al. 2009; Steffen et al. 2015) haben die Autorenkollektive versucht, für wichtige Umweltfelder Grenzwerte für einen sogenannten sicheren Betriebszustand für die Menschheit (safe operating space for humanity) zu definieren. Besonders bekannt geworden ist die Obergrenze von $2{ }^{\circ} \mathrm{C}$ für die globale Erwärmung, da jenseits dieser Grenze unmanageable risks drohen sollen. So hilfreich diese Begrifflichkeiten der planetary boundaries und des safe operating space for humanity sind, um die Dringlichkeit der Problemsituation auch Laien und Politikern verständlich zu machen, so fragwürdig sind sie aus rein wissenschaftlicher Sicht. So hängen die planetary boundaries und der safe operating space for humanity auch von den technologischen Möglichkeiten ab, die uns zur Verfügung stehen und die sich laufend verändern. Darüber hinaus ist der aktuelle Stand der Erdsystemforschung leider noch nicht so weit entwickelt, dass wir für gegebene Technologie-Optionen zuverlässige, belastbare Angaben zu den planetary boundaries und zum safe operating space for humanity machen könnten.

Schließlich sei noch das „One Health-Konzept“ als eine weitere mögliche Zielfunktion für ein modernes Erdsystem-Management genannt (z. B. WHO, FAO, OIE 2019). Diesem liegt die Annahme zugrunde, dass gesunde Menschen nur in einer gesunden Umwelt leben können und dass auch ihre Lebensmittel, ihre Nutztiere und Nutzpflanzen, gesund sein müssen. Dieses Konzept besitzt ebenfalls eine hohe Plausibilität und Überzeugungskraft, lässt sich aber wiederum wissenschaftlich zurzeit noch schwer konkret und operational fassen. Gleichwohl belegt die seit dem Frühjahr 2020 die Welt lähmende Corona-Pandemie die hohe Bedeutung dieser "One-Health"-Zielfunktion, denn das SARS-Cov-2-Virus entstammt - wie etwa auch das Aids-Virus - einer für Tiere harmlosen Zoonose.

Somit sind alle vier genannten möglichen Zielfunktionen für ein ErdsystemManagement (Ökologischer Imperativ, Nachhaltigkeit, Planetare Grenzwerte, One Health) im Kern inhaltlich und kommunikativ sinnvoll und zielführend, im 
konkreten Einzelfall aber (noch) nicht befriedigend wissenschaftlich belastbar und überprüfbar anwendbar. Derzeit liegt die größte wissenschaftliche Stringenz wohl bei dem Konzept der drei Dimensionen der Nachhaltigkeit.

Es bietet sich also an, ein modernes Erdsystemmanagement immer an mehreren Zielfunktionen auszurichten. Wie aber lässt sich ein solches ErdsystemManagement implementieren? Dies setzt mehrere Komponenten voraus. So bedarf es zunächst eines umfassenden Monitorings und Systemverständnisses der zu gestaltenden Umwelt (zum Beispiel einer Küste, eines Flusslaufes, etc.). Ferner muss Einigkeit unter den Stakeholdern über die Zielfunktionen bestehen, wobei auch die Stakeholder global-systemisch gesehen werden müssen. Anschließend müssen die zur Zielerreichung erforderlichen Management-Werkzeuge identifiziert und eingesetzt werden; dabei spielen sowohl technische als auch ökonomische und politische Werkzeuge eine Rolle. Und schließlich bedarf es einer Governance, die den ganzen Prozess des Erdsystem-Managements steuert und überwacht.

Ein derartiges modernes Erdsystem-Management zu entwickeln ist eine riesige Herausforderung und wird Zeit benötigen. Beginnen muss man gleichwohl jetzt. Die Entwicklung der Humanmedizin zu einer modernen, erfolgreichen Wissenschaft hat mehrere Jahrhunderte gebraucht. Die Entwicklung eines modernen Erdsystem-Managements im Sinne einer Heilkunde der Erde muss angesichts der anthropozänen Herausforderung deutlich schneller erfolgen.

\section{Literatur}

Jonas, H. 1984. Das Prinzip Verantwortung: Versuch einer Ethik für die technologische Zivilisation, 1. Aufl., Frankfurt a. M.: Suhrkamp

Rockström, J., W. Steffen, K. Noone, et al. 2009. A safe operating space for humanity. Nature 461: 472-475.

Steffen, W. et al. 2015. Planetary boundaries: Guiding human development on a changing planet. Science 347 (6223).

World Commission on Environment and Development. 1987. Our common future ("Brundtland-Report”). Oxford: Oxford University Press.

Rigaud, Kanta Kumari, de Alex Sherbinin, Bryan Jones, Jonas Bergmann, Viviane Clement, Kayly Ober, Jacob Schewe, Susana Adamo, Brent McCusker, Silke Heuser, und Amelia Midgley. 2018. Groundswell: Preparing for Internal Climate Migration. World Bank, Washington, DC. https://openknowledge.worldbank.org/handle/10986/29461 License: CC BY 3.0 IGO."

World Health Organization (WHO), Food and Agriculture Organization of the United Nations (FAO) and World Organisation for Animal Health (OIE), 2019. Taking a Multisectoral, One Health Approach: A Tripartite Guide to Addressing Zoonotic Diseases in Countries. 
Volker Mosbrugger Prof. Dr. Dr. h.c. Professur am Institut für Geowissenschaften der Goethe-Universität Frankfurt am Main und Generaldirektor der Senckenberg Gesellschaft für Naturforschung (bis Ende 2020), Senckenberganlage 25, 60325 Frankfurt.

volker.mosbrugger@senckenberg.de

Open Access Dieses Kapitel wird unter der Creative Commons Namensnennung 4.0 International Lizenz (http://creativecommons.org/licenses/by/4.0/deed.de) veröffentlicht, welche die Nutzung, Vervielfältigung, Bearbeitung, Verbreitung und Wiedergabe in jeglichem Medium und Format erlaubt, sofern Sie den/die ursprünglichen Autor(en) und die Quelle ordnungsgemäß nennen, einen Link zur Creative Commons Lizenz beifügen und angeben, ob Änderungen vorgenommen wurden.

Die in diesem Kapitel enthaltenen Bilder und sonstiges Drittmaterial unterliegen ebenfalls der genannten Creative Commons Lizenz, sofern sich aus der Abbildungslegende nichts anderes ergibt. Sofern das betreffende Material nicht unter der genannten Creative Commons Lizenz steht und die betreffende Handlung nicht nach gesetzlichen Vorschriften erlaubt ist, ist für die oben aufgeführten Weiterverwendungen des Materials die Einwilligung des jeweiligen Rechteinhabers einzuholen.

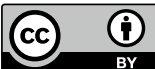

